\title{
Synchronizing Coupled Oscillators in Polygonal Networks with Frustration
}

\author{
Yoko Uwate \\ Dept. of Electrical and Electronic Engineering, \\ Tokushima University \\ 2-1 Minami-Josanjima, Tokushima, Japan \\ Email: uwate@ee.tokushima-u.ac.jp
}

\author{
Yoshifumi Nishio \\ Dept. of Electrical and Electronic Engineering, \\ Tokushima University \\ 2-1 Minami-Josanjima, Tokushima, Japan \\ Email: nishio@ee.tokushima-u.ac.jp
}

\begin{abstract}
In this study, synchronization phenomena observed in coupled polygonal oscillatory networks with frustration is investigated. We focus on the power consumption of coupling resistors of the whole system. By using computer simulations and theoretical analysis, we confirm that the phase differences of the coupled oscillators are solved by finding the minimum value of the power consumption function.
\end{abstract}

\section{INTRODUCTION}

Coupled oscillatory systems are good models to express essential role of high-dimensional nonlinear phenomena occurring in the field of natural sciences. Endo et al. have presented the details of a theoretical analysis and corresponding circuit experiments on electrical circuits oscillators arranged in a ladder, a ring and in a two-dimensional array topology [1]-[3]. Moreover, coupled oscillatory systems can also produce interesting phase patterns, including wave propagation, clustering and complex patterns [4].

On the other hand, there are several types of polygonal network structures (e.g. Honeycomb structure and crystal structure) in the natural science. Generally, for the studies of large-scale network using coupled oscillators, a ring, a ladder and a two dimensional array structure are often investigated. However, there are not many discussions about coupled polygonal oscillatory networks by using electrical oscillators.

In this study, synchronization phenomena in coupled polygonal oscillatory networks with frustration is investigated. In this system, van der Pol oscillators are connected to every corner of polygonal network. First, we consider two identical oscillatory networks are coupled by sharing a branch as a symmetrical system. In this oscillatory system, frustration is occurred by the shared branch. We observe the different synchronization state than the coupled oscillatory system without frustration. We confirm that the phase difference between the shared oscillators is shifted, then other oscillators synchronize to compensate this phase shift. How do the shared oscillators determine this phase shift? What effects will be occurred when such oscillatory system is applied for the large-scale network? In order to make clear these questions, we focus on the power consumption of the coupling resistors in the whole system and investigate the phase difference adjacent oscillators. By using computer simulations and theoretical analysis, we confirm that coupled oscillators tend to synchronize to minimize the power consumption of the whole system. Furthermore, the phase difference of the shared oscillators is solved by finding the minimum value of the power consumption function. Finally, we apply the analysis method of symmetrical system to the asymmetrical and general systems. We expect that the results of this study contribute to understanding of synchronization phenomena observed in general complex networks.

\section{Symmetrical COUPled Oscillatory Networks}

First, we investigate the synchronization phenomena in the symmetrical coupled oscillatory systems. Two identical polygonal oscillatory networks are coupled by sharing a branch as shown in Fig. 1. We call the first and the second oscillators which are connected to both side of polygonal network "shared oscillators."

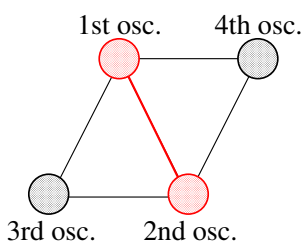

(a) 3-3 coupling network

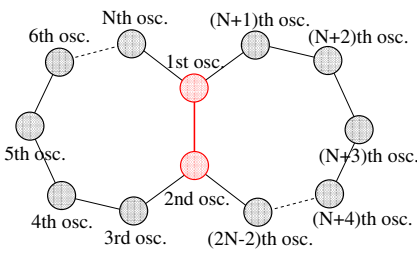

(b) $N-N$ coupling (general) network
Fig. 1. Two coupled polygonal oscillatory networks ( $N$ denotes the number of coupled oscillators connected to the one side of the polygonal oscillatory network).

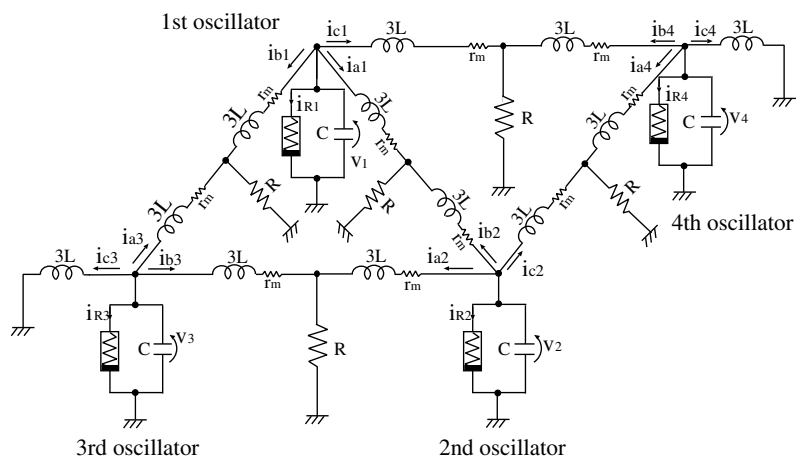

Fig. 2. Circuit model for $3-3$ coupling oscillatory networks.

Next, we develop the expression for the circuit equations of $3-3$ coupling oscillatory networks as shown in Fig. 2. The 
$v_{k}-i_{R k}$ characteristics of the nonlinear resistor are approximated by the following third order polynomial equation,

$$
i_{R k}=-g_{1} v_{k}+g_{3} v_{k}^{3} \quad\left(g_{1}, g_{3}>0\right),(k=1,2,3,4) .
$$

The normalized circuit equations governing the circuit are expressed as

[ $k$ th oscillator]

$$
\left\{\begin{aligned}
& \frac{d x_{k}}{d \tau}=\varepsilon\left(1-\frac{1}{3} x_{k}^{2}\right) x_{k}-\left(y_{a k}+y_{b k}+y_{c k}\right) \\
& \frac{d y_{a k}}{d \tau}=\frac{1}{3}\left\{x_{k}-\eta y_{a k}-\gamma\left(y_{a k}+y_{n}\right)\right\} \\
& \frac{d y_{b k}}{d \tau}=\frac{1}{3}\left\{x_{k}-\eta y_{b k}-\gamma\left(y_{b k}+y_{n}\right)\right\} \\
& \frac{d y_{c k}}{d \tau}=\frac{1}{3}\left\{x_{k}-\eta y_{c k}-\gamma\left(y_{c k}+y_{n}\right)\right\} \\
&(k=1,2,3,4) .
\end{aligned}\right.
$$

In this equations, $\gamma$ is the coupling strength, $\varepsilon$ denotes the nonlinearity of the oscillators and $y_{n}$ denotes the current of neighbor oscillator on coupling resistor.

\section{A. Synchronization Phenomena}

Figure 3 shows the time wave form of the voltage charged at the capacitance of each oscillator obtained from the computer simulation and the circuit experiment. From this figure, we can see that the first and the second oscillators are synchronized at in-phase (phase difference: 0 degree). While, the other combination oscillators synchronize with anti-phase (phase difference: 180 degree).

We did not expect such synchronization state. Because, in the case of three coupled van der Pol oscillators as ring topology, we can observe three-phase synchronization state (phase difference: 120 degree) [5]. So we assumed that two pair of three-phase synchronization could be observed. By adding one more oscillator, the shared oscillators have frustration to synchronize for both side of polygonal networks. In such case, three-phase synchronization could not be observed.

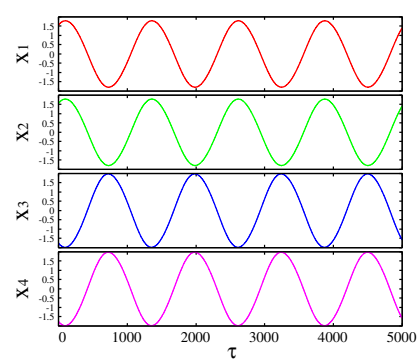

(a) Computer simulation.

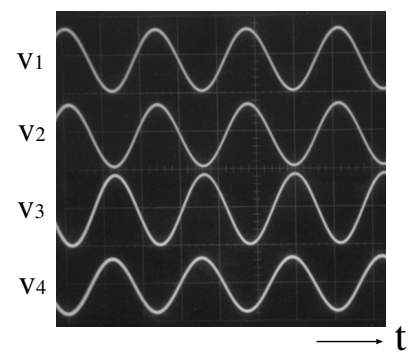

(b) Circuit experiment.
Fig. 3. Time wave form of the voltage charged at the capacitance of each oscillator. (a): $\varepsilon=0.1, \gamma=0.1, \eta=0.0001$, (b): $L=30 \mathrm{mH}, C=33 n F$.

Next, we investigate phase difference of two coupled polygonal oscillators when the number of oscillators in one side of polygonal network $(N)$ are changed from 5 to 11 with odd number of steps. The simulation results are summarized in Table I. $O_{N}$ denotes the total number of oscillators in two coupled polygonal oscillatory system. From this table, we can see that the coupled oscillators do not synchronize with $N$ phase state in each case.

TABLE I

PHASE DIFFERENCE OF SYMMETRICAL OSCILLATORY NETWORKS (COMPUTER SIMULATION)

\begin{tabular}{c|c|c}
\hline \hline Symmetrical Coupling & \multicolumn{2}{|c}{ Phase difference } \\
\cline { 2 - 3 }$N-N\left(O_{N}=2 N-2\right)$ & Shared (1st-2nd) osc. & Other comb. \\
\hline \hline $3-3\left(O_{N}=4\right)$ & 0 & $180^{\circ}$ \\
\hline $5-5\left(O_{N}=8\right)$ & $106^{\circ}$ & $153^{\circ}-154^{\circ}$ \\
\hline $7-7\left(O_{N}=12\right)$ & $130^{\circ}$ & $158^{\circ}-159^{\circ}$ \\
\hline $9-9\left(O_{N}=16\right)$ & $142^{\circ}$ & $162^{\circ}-163^{\circ}$ \\
\hline $11-11\left(O_{N}=20\right)$ & $149^{\circ}$ & $164^{\circ}-165^{\circ}$ \\
\hline
\end{tabular}

\section{B. Power Consumption}

In order to make clear the reason of that $N$-phase synchronization could not be observed in $N-N$ coupling system, we focus on the power consumption of coupling resistors in the whole system. We assume the current of the inductor $(3 L)$ as following equation.

$$
i_{k}(t)=\sqrt{2} I_{e} \sin (\omega t+\varphi) \quad\left(k=1,2,3 \ldots O_{N}\right) .
$$

When the coupling resistance is fixed with $R=1$, the average power consumption of the coupling resistor between $k$ th and $(k+1)$ th oscillators are described as Eq. (4).

$$
P=\frac{1}{2 \pi} \int_{0}^{2 \pi}\left\{i_{k}(t)+i_{k+1}(t)\right\}^{2} d t
$$

We calculate the power consumption of the whole system by using the phase difference obtained from the computer simulations. For comparison, the power consumption of $N$-phase state is also calculated. Table II summarizes the calculation results when $N$ is changed form 3 to 11 . We confirm that the total power consumption of $N$-phase state is larger than the computer simulation results. In the case of 3-3 coupling network (see. Fig. 2), the total number of coupling resistors is five. When the coupled oscillators are synchronized at threephase state, the power consumption of each coupling resistor is $I_{e}{ }^{2}$. Then, the power consumption of the whole system is $5 I_{e}{ }^{2}$ $\left(=5 \times I_{e}{ }^{2}\right)$. While, when the shared oscillators synchronize with in-phase state, the power consumption of shared oscillators is $4 I_{e}^{2}\left(=(I e+I e)^{2}\right)$. And other power consumptions become zero by anti-phase synchronization.

TABLE II

POWER CONSUMPTION FOR SYMMETRICAL OSCILLATORY NETWORKS

\begin{tabular}{c|c|c}
\hline \hline Symmetrical Coupling & \multicolumn{2}{|c}{ Power Consumption } \\
$N-N\left(O_{N}=2 N-2\right)$ & Simulation & $N$-phase presumption \\
\hline \hline $3-3\left(O_{N}=4\right)$ & $4 I_{e}{ }^{2}$ & $5 I_{e}{ }^{2}$ \\
\hline $5-5\left(O_{N}=8\right)$ & $3.132 I_{e}{ }^{2}$ & $3.437 I_{e}{ }^{2}$ \\
\hline $7-7\left(O_{N}=12\right)$ & $2.413 I_{e}{ }^{2}$ & $2.580 I_{e}{ }^{2}$ \\
\hline $9-9\left(O_{N}=16\right)$ & $1.949 I_{e}{ }^{2}$ & $2.050 I_{e}{ }^{2}$ \\
\hline $11-11\left(O_{N}=20\right)$ & $1.639 I_{e}{ }^{2}$ & $1.700 I_{e}{ }^{2}$ \\
\hline
\end{tabular}

From these results, we can see that the coupled oscillators tend to synchronize to minimize the power consumption of the whole system. Then, $N$-phase synchronization can not be obtained in two coupled polygonal oscillatory networks sharing the branch with frustration. 


\section{Theoretical Analysis}

In this section, the phase difference of shared oscillators is solved by using theoretical analysis. We assume two points as following. First, the phase difference between shared oscillators is $\theta$. Second, the phase difference of the other combination oscillators is described by Eq. (5).

$$
\varphi_{N}=\pi-\theta /(N-1)
$$

In the case of $N-N$ coupling system, the power consumption of the whole system is expressed by following equation.

$$
\begin{aligned}
P & =\frac{1}{2 \pi} \int_{0}^{2 \pi}\{\sin \omega t+\sin (\omega t+\theta)\}^{2} d t \\
& +\frac{2(N-1)}{2 \pi} \int_{0}^{2 \pi}\{\sin \omega t \\
& \left.+\sin \left(\omega t+\pi-\frac{\theta}{N-1}\right)\right\}^{2} d t,
\end{aligned}
$$

where the amplitue of current is set to 1 and the coupling resistance is fixed with $R=1$. By integrating Eq. (6), we obtain the equation of the power consumption (Eq. (7)).

$$
P=1+\cos \theta+2(N-1)\{1+\cos (\pi-\theta /(N-1))\} \text {. }
$$

The extreme value is solved by Eq. (8).

$$
\frac{d P}{d \theta}=-\sin \theta+2 \sin (\pi-\theta /(N-1))
$$

When $\frac{d P}{d \theta}=0$ is satisfied, $\theta$ is phase difference of the shared oscillators to minimize the power consumptions of the whole system. Table III summarizes the result of numericallycalculated phase difference by using Eq. (8). We confirm that the value of phase difference obtained from the theoretical analysis has similar value with the computer simulation results (see. Table I).

TABLE III

PHASE DIFFERENCE FOR SYMMETRICAL OSCILLATORY NETWORKS (THEORETICAL ANALYSIS)

\begin{tabular}{c|c|c}
\hline \hline Symmetrical Coupling & \multicolumn{2}{|c}{ Phase difference } \\
\cline { 2 - 3 }$N-N\left(O_{N}=2 N-2\right)$ & Shared (1st-2nd) osc. $(\theta)$ & Other comb. $\left(\varphi_{N}\right)$ \\
\hline \hline $3-3\left(O_{N}=4\right)$ & 0 & $180^{\circ}$ \\
\hline $5-5\left(O_{N}=8\right)$ & $110^{\circ}$ & $152.5^{\circ}$ \\
\hline $7-7\left(O_{N}=12\right)$ & $131^{\circ}$ & $158.2^{\circ}$ \\
\hline $9-9\left(O_{N}=16\right)$ & $142^{\circ}$ & $162.3^{\circ}$ \\
\hline $11-11\left(O_{N}=20\right)$ & $149^{\circ}$ & $165^{\circ}$ \\
\hline
\end{tabular}

As an example, Fig. 4 shows the graphs of Eqs. (7), (8) and the polar representation of the phase differences in the whole system for $5-5$ coupling network (see. Fig. 1 (b)). From Fig. 4 (a), we can see that extreme value of Eq. (7) corresponds to 0 (zero) of Eq. (8). We confirm the the phase space $(4 \pi-\theta)$ is divided equally by the all oscillators other than the shared oscillators (Fig. 4 (b)).

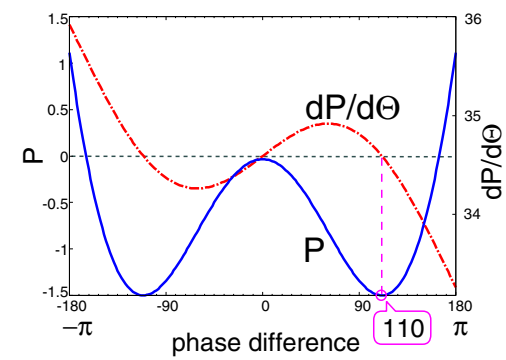

(a) Power consumption.

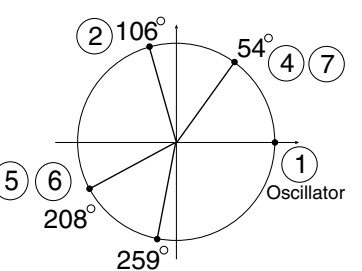

(3) (8)
Fig. 4. Functions of power consumption and polar representation for $5-5$ coupling network.

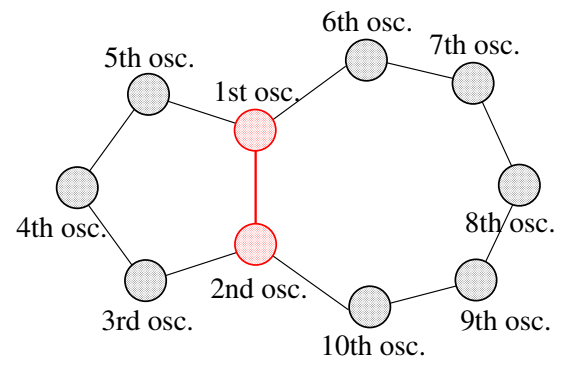

Fig. 5. Asymmetrical coupled polygonal networks for $(N-M)=(5-7)$.

\section{As ymmetrical Coupled Oscillatory Networks}

Next, we consider the asymmetrical system which is composed two different types of polygonal oscillators as shown in Fig. 5.

The phase difference obtained from the computer simulations and the power consumption are summarized in Table IV. By increasing the total coupled number of oscillators, the phase difference of shared oscillators increases and the power consumption becomes small.

TABLE IV

PHASE DIFFERENCE FOR ASYMMETRICAL OSCILLATORY NETWORKS (COMPUTER SIMULATION)

\begin{tabular}{c|c|c|c|c}
\hline \hline $\begin{array}{c}\text { Asymmetrical } \\
\text { Coupling } \\
N-M\end{array}$ & \multicolumn{3}{|c|}{ Phase Difference } & \multirow{2}{*}{ Power } \\
\cline { 2 - 5 } & $\begin{array}{c}\text { Shared } \\
\text { osc. }(\theta)\end{array}$ & $\begin{array}{c}N \text { osc. } \\
\left(\varphi_{N}\right)\end{array}$ & $\begin{array}{c}M \text { osc. } \\
\left(\varphi_{M}\right)\end{array}$ & \\
\hline \hline $3-5$ & $67^{\circ}$ & $145^{\circ}-146^{\circ}$ & $163^{\circ}-164^{\circ}$ & 3.787 \\
\hline $3-7$ & $86^{\circ}$ & $136^{\circ}-137^{\circ}$ & $166^{\circ}-167^{\circ}$ & 3.593 \\
\hline $3-9$ & $94^{\circ}$ & $132^{\circ}-133^{\circ}$ & $168^{\circ}-169^{\circ}$ & 3.474 \\
\hline $3-11$ & $102^{\circ}$ & $129^{\circ}-130^{\circ}$ & $171^{\circ}-172^{\circ}$ & 3.329 \\
\hline $5-7$ & $119^{\circ}$ & $150^{\circ}-151^{\circ}$ & $160^{\circ}-161^{\circ}$ & 2.800 \\
\hline $5-9$ & $125^{\circ}$ & $160^{\circ}-161^{\circ}$ & $164^{\circ}-165^{\circ}$ & 2.608 \\
\hline $5-11$ & $129^{\circ}$ & $147^{\circ}-148^{\circ}$ & $165^{\circ}-166^{\circ}$ & 2.484 \\
\hline $7-9$ & $136^{\circ}$ & $158^{\circ}-159^{\circ}$ & $163^{\circ}-164^{\circ}$ & 2.191 \\
\hline $7-11$ & $140^{\circ}$ & $156^{\circ}-157^{\circ}$ & $165^{\circ}-166^{\circ}$ & 2.046 \\
\hline
\end{tabular}

Next, the phase difference of shared oscillators is solved by using same as previous theoretical analysis. For the case of the asymmetric system, we calculate the power consumption of each polygonal network separately. The equations of the total power consumption is described as follows.

$$
\begin{aligned}
P & =1+\cos \theta+(N-1)\{1+\cos (\pi-\theta /(N-1))\} \\
& +(M-1)\{1+\cos (\pi-\theta /(M-1))\} .
\end{aligned}
$$




$$
\begin{aligned}
\frac{d P}{d \theta} & =-\sin \theta+\sin (\pi-\theta /(N-1)) \\
& +\sin (\pi-\theta /(M-1)) .
\end{aligned}
$$

Table V shows the numerically-calculated phase difference by using Eq. (10). From this table, we can see that the results of the theoretical analysis match well with simulation results (see. Table IV).

TABLE V

PHASE DIFFERENCE FOR ASYMMETRICAL OSCILLATORY NETWORKS (THEORETICAL ANALYSIS)

\begin{tabular}{c|c|c|c}
\hline \hline Asymmetrical & \multicolumn{3}{|c}{ Phase Difference } \\
\cline { 2 - 4 }$N-M$ & Shared osc. $(\theta)$ & $N$ osc. $\left(\varphi_{N}\right)$ & $M$ osc. $\left(\varphi_{M}\right)$ \\
\hline \hline $3-5$ & $79^{\circ}$ & $140.5^{\circ}$ & $160.3^{\circ}$ \\
\hline $3-7$ & $93^{\circ}$ & $133.5^{\circ}$ & $164.5^{\circ}$ \\
\hline $3-9$ & $100^{\circ}$ & $130.0^{\circ}$ & $167.5^{\circ}$ \\
\hline $3-11$ & $104^{\circ}$ & $149.8^{\circ}$ & $159.8^{\circ}$ \\
\hline $5-7$ & $121^{\circ}$ & $128.0^{\circ}$ & $169.6^{\circ}$ \\
\hline $5-9$ & $127^{\circ}$ & $148.3^{\circ}$ & $164.1^{\circ}$ \\
\hline $5-11$ & $130^{\circ}$ & $147.5^{\circ}$ & $167.0^{\circ}$ \\
\hline $7-9$ & $136^{\circ}$ & $157.3^{\circ}$ & $163.0^{\circ}$ \\
\hline $7-11$ & $140^{\circ}$ & $156.7^{\circ}$ & $166.0^{\circ}$ \\
\hline
\end{tabular}

\section{Application for General Networks}

Finally, we apply the previous theoretical approach to solve the phase difference of shared branch to the general networks. One example of general coupled polygonal network is shown in Fig. 6.

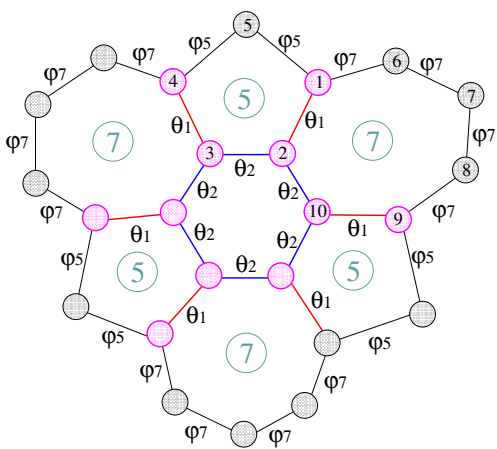

Fig. 6. One example of general coupled oscillatory networks.

For this system, first, we define the two phase differences $\theta_{1}$ and $\theta_{2}$ as shown in Fig. 6. Then we can obtain the phase differences $\varphi_{5}$ and $\varphi_{7}$ as described by the following equation.

$$
\varphi_{N_{p}}=\frac{\left(N_{p}-1\right) \pi-\left(2 \theta_{1}+\theta_{2}\right)}{N_{p}-3} \quad\left(N_{p}=5,7\right) .
$$

The power consumption equation is expressed as

$$
\begin{aligned}
P & =6\left(2 \pi+\pi \cos \theta_{1}\right)+6\left(2 \pi+\pi \cos \theta_{2}\right) \\
& +6\left(2 \pi+\frac{\left(4 \pi-\left(2 \theta_{1}+\theta_{2}\right)\right)}{2}\right) \\
& +12\left(2 \pi+\frac{\left(6 \pi-\left(2 \theta_{1}+\theta_{2}\right)\right)}{4}\right) .
\end{aligned}
$$

Figure 7 shows the function of power consumption with $3 \mathrm{D}$ plot when $\theta_{1}$ and $\theta_{2}$ are changed from $-\pi$ to $\pi$. By calculating this function, we obtain $\theta_{1}$ and $\theta_{2}$ which show the minimum value of this function. In the range of $[0: \pi]$, the minimum value of $\theta_{1}$ and $\theta_{2}$ are $130.5^{\circ}$ and $158.0^{\circ}$, respectively.

Table VI summarizes the phase differences obtained from the computer simulations and the theoretical analysis using Eqs. (11), (12). We confirm that the phase differences of the theoretical analysis match pretty well to the results of the computer simulations. We consider that even if calculation becomes more complex for the asymmetrical system, the same theoretical analysis can be applied.

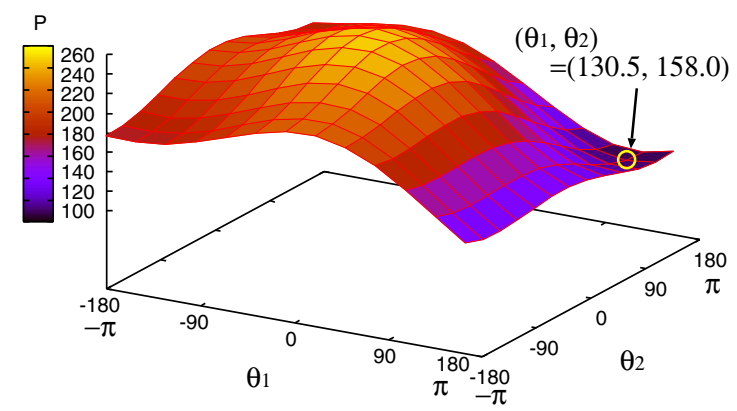

Fig. 7. Power function of Eq. (12).

TABLE VI

PHASE DIFFERENCE FOR GENERAL OSCILLATORY NETWORKS

\begin{tabular}{c|c|c}
\hline \hline \multirow{2}{*}{ Phase Type } & \multicolumn{2}{|c}{ Phase Difference } \\
\cline { 2 - 3 } & Simulation & Theory \\
\hline \hline$\theta_{1}$ & $130^{\circ}$ & $130.5^{\circ}$ \\
\hline$\theta_{2}$ & $158^{\circ}$ & $158.0^{\circ}$ \\
\hline$\varphi_{5}$ & $150.7^{\circ}$ & $150.5^{\circ}$ \\
\hline$\varphi_{7}$ & $164.5^{\circ}$ & $165.3^{\circ}$ \\
\hline
\end{tabular}

From these results, we can apply this theoretical analysis for the large-scale networks close to the real networks existing in the our living world.

\section{CONCLUSION}

In this study, we have investigated synchronization phenomena in coupled polygonal oscillatory networks with frustration. By using computer simulations and theoretical analysis, we have confirmed that coupled oscillators tend to synchronize to minimize the power consumption of whole system. Investigation of synchronization phenomena observed in coupled chaotic oscillator in the polygonal networks with frustration is our future work.

\section{REFERENCES}

[1] T. Endo and S. Mori, "Mode analysis of a multimode ladder oscillator," IEEE Trans. Circuits Syst., vol.23, pp.100-113, Feb. 1976.

[2] T. Endo and S. Mori, "Mode analysis of two-dimensional low-pass multimode oscillator," IEEE Trans. Circuits Syst., vol.23, pp.517-530, Sep. 1976

[3] T. Endo and S. Mori, "Mode analysis of a ring of a large number of mutually coupled van der Pol oscillators," IEEE Trans. Circuits Syst., vol.25, no.1, pp.7-18, Jan. 1978.

[4] M. Yamauchi, Y. Nishio and A. Ushida, "Phase-waves in a ladder of oscillators" IEICE Trans. Fundamentals, vol.E86-A, no.4, pp.891-899, Apr. 2003.

[5] Y. Uwate, Y. Nishio and R. Stoop, "Synchronization in Three Coupled van der Pol Oscillators with Different Coupling Strength," Proc. of NCSP'10, pp. 109-112, Mar. 2010. 